$\xi=-$

\title{
On the Nordtvedt effect in Minkowski spacetime with nonlinear connection
}

\author{
Kostadin Trenčevski ${ }^{1}$, Emilija Celakoska ${ }^{2 *}$ \\ ${ }^{1}$ Faculty of Natural Sciences and Mathematics, Ss. Cyril and Methodius University in Skopje, Republic of Macedonia \\ ${ }^{2}$ Faculty of Mechanical Engineering, Ss. Cyril and Methodius University in Skopje, Republic of Macedonia \\ *Corresponding author E-mail:emilija.celakoska@mf.edu.mk
}

\begin{abstract}
The Lunar Laser Ranging (LLR) experiment provided precise data which brought the possibility to make more stringent conclusions for the foundations of gravitational theories, i.e. the Equivalence Principles. Beside some effects of non - gravitational origin, the LLR data was fitted with the well-known gravitational effects such as the apsidal and geodetic precessions, the time delay, etc. The Nordtvedt effect in General Relativity (GR) vanishes, while the LLR experiment data of the Earth-Moon distance and the laboratory experiments with experimental bodies made of different chemical compositions measured a variation of distance in millimeters. According to the mathematical model of gravitation in Minkowski space endowed with a nonlinear connection we obtained a result closer to the experimental measurements. More precisely, we obtained a difference of $0.17 \mathrm{~mm}$ (or $0.28 \mathrm{~mm}$, depending on the value of the scaling factor) from the LLR measurements of the variation of the Earth-Moon distance, while the corresponding result in GR makes a difference from the LLR measurements of $5.7 \mathrm{~mm}$. The gravitational theory with nonlinear connection in Minkowski space gives the same results for the confirmed GR effects, nevertheless it yields some additional variations of the distance concerning the Nordtvedt effect.
\end{abstract}

Keywords: lunar laser ranging; equivalence principles; Earth-Moon distance, gravitation.

\section{Introduction}

Advances in the understanding of the gravitation are significantly accomplished by the feedback between the experimental data and the theoretical framework. Lunar laser ranging (LLR) experiment is one of the most precise experiments and it measured the possible difference between the Earth's and Moon's acceleration toward the Sun, i.e. the Nordtvedt effect. The calculation of the Nordtvedt effect in the metric theories is presented in [4], [11], [12] and it is obtained by summation of several gravitational non-zero effects. The Nordtvedt effect is a measure of the Equivalence principle and a possible non-zero part of the Moon's equations of motion relative to the Earth, considering the Sun-Earth-Moon system. It is defined by the fractional difference in acceleration between the Earth and the Moon towards the Sun, which is the quantity called the Eötvös ratio. The value of this quantity in the theory of GR is zero.

The analytical and numerical techniques on the LLR data improved the accuracy of the experimental results. Thus, the theories of gravitation are provided with a reliable tool for inspection with respect to the Nordtvedt effect. The difference between the LLR experimental results [13] and the GR theoretical value, including the laboratory experimental result [1], is $5.7 \mathrm{~mm}$.

In this paper we present a theoretical result of the Nordtvedt effect which gives value closer to the precise measurements, i.e. a displacement which deviates from the LLR measurements no more than $0.28 \mathrm{~mm}$ (or $0.17 \mathrm{~mm}$, depending on the value of the scaling factor). The mathematical model presented in [8] and applied in [9] and [10] is based on a nonlinear connection (nonlinearity of the covariant derivative) in the Minkowski flat space. Note that the metric theories use the ample Riemann differential-geometric apparatus for manifolds and then in the weak field reduce to differential equations in terms of the lowest-order deviations from the Newton's laws. Unlike the metric theories, this approach implements coordinate transformation of a specific field tensor by the well-known Lorentz boost link tensor, so that a given 4-vector of velocity can be directly parallel-transported. The field tensor is obtained from a scalar gravitational potential and the 4-vector of velocity related to the body which contribute to the gravitational field. The equations of motion follow straightforwardly and the calculations of the experimentally confirmed gravitational effects give identical results as the theory of GR in the weak field. The advantage of this theoretical approach is the simplicity of the modeling and calculation of the effects. In fact, the direct correspondence with the observable physical quantities, as it is the case in the classical physics, makes it very convenient in practice.

The plan of the paper is as follows. In section 2 we present a scalar potential with an unknown parameter which is implemented in the field tensor. We form the (nonlinear) parallel transport and the corresponding equations of motion. The most reliable effects in gravitational physics are the orbital mechanical effects, such as the apsidal precession. Thus, using these equations of motion we derive the apsidal precession of the orbits of two bodies with significant masses, orbiting around their center of gravity. The obtained formula is of the form of the well-known periastron precession of binaries in GR. Let us note that the case of perihelion precession does not require parameters for the potential in this approach, but it gives the correct result [8] according to the experimental data and also agrees with the theoretical result in GR. However, in the case of two masses, by comparison of coefficients, we are able to 
find the unknown parameter for the potential and have definite expressions for the field tensor.

In section 3 we apply the derived acceleration for the case of the Earth and the Moon in the fractional difference of accelerations. However, we do not include the apsidal motions in the sum of the effects contributing to the Nordtvedt effect. Namely, since the perigee and the perihelion precession difference relative to the Sun is already used in the fitting of the LLR data, i.e. it is included in the final estimation of the Nordtvedt effect in [14], we use that estimation as a reference for comparison. In the matter of apsidal motions and their contributions expressed in the post-Newtonian order of approximation in the Newton-type of equations of motion, this mathematical model is in complete agreement with the theory of GR, generating the corresponding expressions rather directly. Next, in section 3, a gravitational effect of deformation of the Moon's orbit around the Earth is considered. Finally, we conclude that the sum of three values (along the distance vector) contribute to the Nordtvedt effect: the laboratory measured effect which is caused by different chemical compositions of objects, the ratio using the accelerations obtained in the presented theoretical approach in section 2 and the effect of deformation of orbits for gravitationally bound objects.

\section{Theoretical considerations of nonlinear parallel transport}

Firstly, we consider two bodies with masses $M$ and $m$ in a binary gravitational system, orbiting around a common barycentre. We choose a coordinate system having an origin at the barycentre of the two bodies and let its 4 -vector of velocity be $\left(W_{i}\right)=(0,0,0,1)$. We denote the 4-vectors of velocity of the body with mass $M$ and the body with mass $m$ by $\left(U_{i}\right)=\left(1-\frac{u^{2}}{c^{2}}\right)^{-\frac{1}{2}}\left(\frac{u_{x}}{i c}, \frac{u_{y}}{i c}, \frac{u_{z}}{i c}, 1\right)$ and $\left(V_{i}\right)=\left(1-\frac{v^{2}}{c^{2}}\right)^{-\frac{1}{2}}\left(\frac{v_{x}}{i c}, \frac{v_{y}}{i c}, \frac{v_{z}}{i c}, 1\right)$, with respect to the barycentre correspondingly, where the corresponding 3-vectors of velocity are $\boldsymbol{u}=\left(u_{x}, u_{y}, u_{z}\right)$ and $\boldsymbol{v}=\left(v_{x}, v_{y}, v_{z}\right)$, with norms $u$ and $v$. Since the gravitational effects that we will consider take place in no more than two dimensions, it is sufficient to denote the coordinates of the body with mass $m$ by $(x, y, 0)$ and the coordinates of the body with mass $M$ by $\left(x^{*}, y^{*}, 0\right)$. Then, the corresponding velocities of the bodies would be $\boldsymbol{u}=\left(u_{x}, u_{y}, 0\right)$ and $\boldsymbol{v}=\left(v_{x}, v_{y}, 0\right)$, and the acceleration and the angular velocity of the body with mass $m$ would be $\boldsymbol{a}=\left(a_{x}, a_{y}, 0\right)$ and $\boldsymbol{\omega}=\left(0,0, \omega_{z}\right)$. Let the general form of the gravitational potential be

$\varphi=-\left(1+\frac{n \cdot G m}{R c^{2}}\right) \frac{M c^{2}}{M-m} \ln \frac{1+\frac{G M}{R c^{2}}}{1+\frac{G m}{R c^{2}}}$,

where $R$ is the norm of the vector $\boldsymbol{R}$ from the body with mass $M$ to the body with mass $m$ and $n$ is an unknown parameter which can be determined by experimental measurements of the evident mechanical gravitational effects of post-Newtonian order like the apsidal motion of orbits. The aim is to derive the Earth's and Moon's acceleration towards the Sun and their fractional difference by using one potential and having in mind that the three bodies form the system and not the two pairs Sun-Earth and SunMoon separately. For the analysis in our Solar system it is sufficient to use the following approximation of the potential (1),

$$
\varphi=-\left(1+\frac{n \cdot G m}{R c^{2}}\right) \frac{M c^{2}}{M-m} \ln \left(1+\frac{G(M-m)}{R c^{2}}\right) .
$$

Now, let us construct a field tensor $\phi$ using the 4-vector of velocity $\left(U_{i}\right)$ of the body with mass $M$ and the gradient $\nabla \varphi=\left(\frac{\partial \varphi}{\partial x}, \frac{\partial \varphi}{\partial y}, \frac{\partial \varphi}{\partial z}\right)$. The body with mass $M$ influences the trajectory of the body with mass $m$ and, correspondingly, the symmetrical case is the other way around. Thus, it is expected that the field tensor which will be used to derive the equations of motion for the body with mass $m$ to include the velocity $\left(U_{i}\right)$. Analogously to the Lienard-Wiechert potentials in electromagnetism, let the relations among the elements of the tensor $\phi$ be the following:

$$
\begin{aligned}
\frac{1}{i}\left(\varphi_{32}, \varphi_{13}, \varphi_{21}\right)= & \frac{1}{R} \boldsymbol{R} \times\left(\varphi_{41}, \varphi_{42}, \varphi_{43}\right), \\
c^{2}\left(\varphi_{41}, \varphi_{42}, \varphi_{43}\right)= & -G M\left(R-\frac{\boldsymbol{R} \cdot \boldsymbol{u}}{c}\right)^{-3}\left[\left(\boldsymbol{R}-\frac{\boldsymbol{u}}{c} R\right)\right. \\
& \left.+\frac{1}{c^{2}} \boldsymbol{R} \times\left(\left(\boldsymbol{R}-\frac{\boldsymbol{u}}{c} R\right) \times \frac{\partial \boldsymbol{u}}{\partial t^{*}}\right)\right],
\end{aligned}
$$

assuming that the gravitational interaction from the body with mass $M$ at the space-time point $\left(x^{*}, y^{*}, z^{*}, i c t^{*}\right)$ arrives to the space-time point $(x, y, z, i c t)$, after time $t-t^{*}$. This means that $t^{*}$ is a solution of the equation $t=t^{*}+\frac{R\left(t^{*}\right)}{c}$. Since in case of twobody problem $\frac{\partial \boldsymbol{u}}{\partial t^{*}}$ is collinear with $\boldsymbol{R}$ and neglecting the terms of order $c^{-3}$, we can replace $\frac{\partial \boldsymbol{u}}{\partial t^{*}}=0$. Then, the second equation in (3) reduces to

$$
c^{2}\left(\varphi_{41}, \varphi_{42}, \varphi_{43}\right)=-\frac{G M}{R^{3}} \boldsymbol{R} \frac{1-\frac{u^{2}}{c^{2}}}{\left(1-\frac{u^{2}}{c^{2}} \sin ^{2} \theta\right)^{3 / 2}},
$$

where $\theta$ is the angle between $\boldsymbol{R}$ and $\boldsymbol{u}$. The coefficient $\left(1-\frac{u^{2}}{c^{2}}\right)\left(1-\frac{u^{2}}{c^{2}} \sin ^{2} \theta\right)^{-3 / 2}$ can be approximated up to $c^{-2}$ to $\left(1-\frac{u^{2}}{c^{2}}\right)^{-1 / 2}\left(1+\frac{1}{c^{2}} \frac{m^{2}}{(M+m)^{2}}\left(\frac{d R}{d t}\right)^{2}\right)^{-3 / 2}$. Nevertheless, the second multiplier of this expression cannot contribute to the apsidal motion and thus, for our purposes, we will not consider it. According to (3), i.e. (4) and the latter argument, we construct the elements of the tensor $\phi$ as follows,

$$
\begin{aligned}
& \phi_{41}=-\phi_{14}=-\frac{1}{c^{2}} U_{4} \frac{\partial \varphi}{\partial x}, \phi_{32}=-\phi_{23}=-\frac{1}{c^{2}}\left(U_{3} \frac{\partial \varphi}{\partial y}-U_{2} \frac{\partial \varphi}{\partial z}\right), \\
& \phi_{42}=-\phi_{24}=-\frac{1}{c^{2}} U_{4} \frac{\partial \varphi}{\partial y}, \phi_{13}=-\phi_{31}=-\frac{1}{c^{2}}\left(U_{1} \frac{\partial \varphi}{\partial z}-U_{3} \frac{\partial \varphi}{\partial x}\right), \\
& \phi_{43}=-\phi_{34}=-\frac{1}{c^{2}} U_{4} \frac{\partial \varphi}{\partial z}, \phi_{21}=-\phi_{12}=-\frac{1}{c^{2}}\left(U_{2} \frac{\partial \varphi}{\partial x}-U_{1} \frac{\partial \varphi}{\partial y}\right) .
\end{aligned}
$$

In order to correlate the tensor $\phi$ with the physical reality, it is almost obvious that the fourth row (column) with the gradient of the potential could represent the acceleration $\boldsymbol{a}$, while the spatial 
part could be related to the angular velocity $\boldsymbol{\omega}$. Thus, we can write the physical interpretation of $\phi$ as

$\phi=\left[\begin{array}{cccc}0 & -i \omega_{z} / c & i \omega_{y} / c & -a_{x} / c^{2} \\ i \omega_{z} / c & 0 & -i \omega_{x} / c & -a_{y} / c^{2} \\ -i \omega_{y} / c & i \omega_{x} / c & 0 & -a_{z} / c^{2} \\ a_{x} / c^{2} & a_{y} / c^{2} & a_{z} / c^{2} & 0\end{array}\right]$,

where according to (5), now we have

$$
\begin{aligned}
& a_{x}=-U_{4} \frac{\partial \varphi}{\partial x}, \quad a_{y}=-U_{4} \frac{\partial \varphi}{\partial y}, \quad a_{z}=-U_{4} \frac{\partial \varphi}{\partial z}, \\
& \omega_{x}=\frac{i}{c}\left(U_{3} \frac{\partial \varphi}{\partial y}-U_{2} \frac{\partial \varphi}{\partial z}\right), \quad \omega_{y}=\frac{i}{c}\left(U_{1} \frac{\partial \varphi}{\partial z}-U_{3} \frac{\partial \varphi}{\partial x}\right), \\
& \omega_{z}=\frac{i}{c}\left(U_{2} \frac{\partial \varphi}{\partial x}-U_{1} \frac{\partial \varphi}{\partial y}\right) .
\end{aligned}
$$

We calculate these elements by approximating up to $c^{-2}$ and using that $r \approx \frac{M}{M+m} R$, where $r=\sqrt{x^{2}+y^{2}}$, we obtain

$a_{x}=-\left(1-\frac{u^{2}}{c^{2}}\right)^{-\frac{1}{2}} \frac{G M}{R^{2}+\frac{G(\alpha M+\beta m) R}{c^{2}}} \frac{x}{r}$,

$a_{y}=-\left(1-\frac{u^{2}}{c^{2}}\right)^{-\frac{1}{2}} \frac{G M}{R^{2}+\frac{G(\alpha M+\beta m) R}{c^{2}}} \frac{y}{r}$,

where $\alpha$ and $\beta$ depend on the value of $n$ in the potential (2). Now, $a_{z}=0=\omega_{x}=\omega_{y}$ for effects in no more than two dimensions and using the approximations $u_{x}=-v_{x} \frac{m}{M}$ and $u_{y}=-v_{y} \frac{m}{M}$, we obtain

$\omega_{z}=\left(1-\frac{u^{2}}{c^{2}}\right)^{-\frac{1}{2}} \frac{G m}{R^{2}+\frac{G(\alpha M+\beta m) R}{c^{2}}} \frac{v_{x} y-v_{y} x}{r c^{2}}$.

Note that similar correlation of the field tensor with the physical reality is successfully implemented in the electromagnetism for the electromagnetic tensor where the Lienard-Wiechert potentials are used. However, the resemblance owes only to the tendency of the nature to form its laws in the Stokes' theorem pattern of conservation of quantities. Still, unlike the theory of electromagnetism, the case of the gravitational interaction is significantly compounded by transformation of the tensor $\phi$ and the given 4velocity by Lorentz boost link.

The Lorentz boost link was independently found by several authors [2], [3], [7] and we present it here in the form

$$
P_{i j}=\delta_{i j}-\frac{1}{1+W_{s} V_{s}}\left(V_{i} V_{j}+V_{i} W_{j}+W_{i} V_{j}+W_{i} W_{j}\right) .
$$

The tensor $P_{i j}$ is such that $P_{i j} W_{j}=V_{j}$ i.e., it transforms the velocity $\left(W_{i}\right)$ of the barycentre to the velocity $\left(V_{i}\right)$ of the body with mass $m$. Now, the parallel transport (connection) is given by the tensor $P^{T} \phi P$, so the equations of motion of the body with mass $m$ are $\frac{d V_{i}}{d s}=P_{r i} \phi_{r k} P_{k j} V_{j}$

These equations of motion (and then the apsidal precession) are calculated in analogous manner as it is made in [8] (section 4), however now with unknown coefficients $\alpha$ and $\beta$ which are functions of the parameter $n$. It should be noted that therein (section 3.1) the calculations for perihelion shift, using $m$ as negligible mass, are self-contained and give the same result as in GR, i.e. $\Delta \psi=\frac{6 \pi\left(4 \pi^{2}\right)^{1 / 3} G^{2 / 3}}{T^{2 / 3} c^{2}\left(1-\varepsilon^{2}\right)} M^{2 / 3}$. From (9), in the case of two masses, somewhat tedious, but straightforward derivation of the apsidal precession of the orbit gives

$\Delta \psi=\frac{(7-\alpha) M+(1-\beta) m}{M+m} \frac{\pi\left(4 \pi^{2}\right)^{1 / 3} G^{2 / 3}}{T^{2 / 3} c^{2}\left(1-\varepsilon^{2}\right)}(M+m)^{2 / 3}$,

where $T$ is the orbital period and $\varepsilon$ is the eccentricity of the considered orbit. Since the derivation of the angle $\Delta \psi$ is symmetric with respect to the orbit of the each of the bodies with masses $M$ and $m$, in (10) should be satisfied $7-\alpha=1-\beta$. Thus, $\alpha$ and $\beta$ are determined by the fact that

$\Delta \psi=\frac{6 \pi\left(4 \pi^{2}\right)^{1 / 3} G^{2 / 3}}{T^{2 / 3} c^{2}\left(1-\varepsilon^{2}\right)}(M+m)^{2 / 3}$,

i.e. $\alpha=1, \beta=-5$. So, for these values of $\alpha$ and $\beta$ the equations of motion (9), for $i=1$ and $i=2$, take their definite form

$$
\begin{aligned}
\frac{d^{2} x}{d t^{2}}= & -\left(1-\frac{u^{2}}{c^{2}}\right)^{-\frac{1}{2}}\left(1+\frac{v^{2}}{c^{2}}\right) \frac{G M}{R^{2}+\frac{G(M-5 m) R}{c^{2}} \frac{x}{r}} \\
& +\frac{5}{2} \frac{v_{x}}{c^{2}} \frac{G M}{R^{2}} \frac{x v_{x}+y v_{y}}{r}-2 \frac{v_{y}}{c^{2}} \frac{G m}{R^{2}} \frac{x v_{y}-y v_{x}}{r}, \\
\frac{d^{2} y}{d t^{2}}= & -\left(1-\frac{u^{2}}{c^{2}}\right)^{-\frac{1}{2}}\left(1+\frac{v^{2}}{c^{2}}\right) \frac{G M}{R^{2}+\frac{G(M-5 m) R}{c^{2}} \frac{y}{r}} \\
& +\frac{5}{2} \frac{v_{y}}{c^{2}} \frac{G M}{R^{2}} \frac{x v_{x}+y v_{y}}{r}-2 \frac{v_{x}}{c^{2}} \frac{G m}{R^{2}} \frac{x v_{y}-y v_{x}}{r}
\end{aligned}
$$

The acceleration (7) with the values $\alpha=1, \beta=-5$ will be used in the next section. It is worthwhile noting that these values correspond to the "correcting multiplier" $-\left(1+\frac{3 G m}{R c^{2}}\right)$ in (1), i.e. the scalar potential is $\varphi=-\left(1+\frac{3 G m}{R c^{2}}\right) \frac{M c^{2}}{M-m} \ln \frac{1+\frac{G M}{R c^{2}}}{1+\frac{G m}{R c^{2}}}$.

\section{On the Nordtvedt effect}

The results of the Nordtvedt effect measurements are given and reviewed in [13], [14] and [15]. According to the LLR data [13],

$\frac{a_{e}-a_{m}}{\left(a_{e}+a_{m}\right) / 2}=(-0.96 \pm 1.42) \times 10^{-13}$ 
where $a_{e}$ and $a_{m}$ are the magnitudes of the accelerations of the Earth and the Moon towards the Sun, respectively. This Eötvös ratio corresponds to the change of the distance Earth-Moon [13] (Table 2)

$$
\Delta r=(2.8 \pm 4.1) \cos D[\mathrm{~mm}]
$$

using the proportionality coefficient $-2.9 \times 10^{13} \mathrm{~mm}$, [6]. This value has been corrected for the solar radiation pressure. The value (12), i.e. (13) is measured by the LLR experiment, thus it is a referent value for comparison among theories.

The effect (13) represents a sum of three effects in the presented approach. The first effect, which must be incorporated in the fitting of the Nordtvedt effect, is caused by the different chemical compositions of the Earth and the Moon and it is laboratory measured. According to [1], the Eötvös ratio for this effect is

$$
\frac{a_{e}-a_{m}}{\left(a_{e}+a_{m}\right) / 2}=(1.0 \pm 1.4) \times 10^{-13} .
$$

This corresponds to the variation of distance of

$$
\Delta r=-(2.9 \pm 4.06) \cos D[\mathrm{~mm}]
$$

The second effect contributing to the Nordtvedt effect is the fractional difference of accelerations involving the magnitude of the acceleration obtained in (7), where the distance from the barycentre from each of the considered bodies to the Sun now can be neglected. We have

$a_{e}-a_{m}=\frac{G M}{R^{2}}\left(1+\frac{\left(5 m_{e}-M\right) G}{R c^{2}}\right)-\frac{G M}{R^{2}}\left(1+\frac{\left(5 m_{m}-M\right) G}{R c^{2}}\right)$

and hence,

$$
\frac{a_{e}-a_{m}}{\left(a_{e}+a_{m}\right) / 2}=5 \frac{\left(m_{e}-m_{m}\right) G}{R c^{2}}=1.484 \times 10^{-13}
$$

where $m_{e}, m_{m}$ and $M$ are the masses of the Earth, the Moon and the Sun respectively, and $R$ is the distance to the Sun. This corresponds to the variation of distance of

$$
\Delta r=-4.3036 \cos D[\mathrm{~mm}] .
$$

Now, for the third contributing effect let $r_{1}$ be the distance EarthMoon for $\cos D=-1$ and let $r_{2}$ be the distance Earth-Moon for $\cos D=1$, neglecting the variation of distance by given by the previous contributions (13) and (14) to the effect, and neglecting also the large discrepancy caused by the tidal accelerations [11]. Let us denote by $x, y, z, t$ the coordinates associated to the Earth and let $X, Y, Z, T$ be the coordinates near the Moon, observed from the Earth. We assume that the unperturbed trajectory of the Earth around the Sun is circular and then, without loss of generality, $x, y$, $z$ and $t$ can be the coordinates associated to an observer far from massive objects. Since the distances are correlated to the mass generating the field, it is sufficient to use the coordinate change $(d X, d Y, d Z)=\mu(d x, d y, d z)$ and $d T=\mu^{-1} d t$, where $\mu=1+\frac{G M}{R c^{2}}$.

Let $R$ be the distance Moon-Sun and $R_{0}$ be the distance EarthSun. We obtain $\left(\frac{d X}{d T}, \frac{d Y}{d T}, \frac{d Z}{d T}\right)=\mu^{2}\left(\frac{d x}{d t}, \frac{d y}{d t}, \frac{d z}{d t}\right)$, where we can approximate
$\mu=1+\frac{G M}{R c^{2}}=1+\frac{G M}{\left(R_{0}+y\right) c^{2}} \approx\left(1+\frac{G M}{R_{0} c^{2}}\right)\left(1-y \frac{G M}{R_{0}^{2} c^{2}}\right)$.

Now, let us replace $\mu=C(1+k y)$, where $k=-\frac{G M}{R_{0}^{2} c^{2}}$ and $C=1+\frac{G M}{R_{0} c^{2}}$. Let the Moon be between the Sun and the Earth at $t=t_{0}$, i.e. $\cos D=1$, also let $\cos D=0$ at $t=t_{1}$, and let the Earth be between the Sun and the Moon at $t=t_{2}$, i.e. $\cos D=-1$. Then the required distances observed geometrically, up to $c^{-2}$, are given by

$$
\begin{aligned}
r_{2} & =\int_{t_{0}}^{t_{1}} \frac{d Y}{d T} d t=\int_{t_{0}}^{t_{1}} \frac{d y}{d t} C^{2}(1+k y)^{2} d t=\left.C^{2}\left(y+k y^{2}\right)\right|_{t_{0}} ^{t_{1}} \\
& =C^{2}\left[0-(-r)+k\left(0^{2}-(-r)^{2}\right)\right]=C^{2}\left(r-k r^{2}\right) \\
r_{1} & =\int_{t_{1}}^{t_{2}} \frac{d Y}{d T} d t=\int_{t_{1}}^{t_{2}} \frac{d y}{d t} C^{2}(1+k y)^{2} d t=\left.C^{2}\left(y+k y^{2}\right)\right|_{t_{1}} ^{t_{2}} \\
& =C^{2}\left[r-0+k\left(r^{2}-0^{2}\right)\right]=C^{2}\left(r+k r^{2}\right),
\end{aligned}
$$

where we assumed that, apart from the tidal acceleration in orthonormal coordinates, the Moon's trajectory around the Earth is a circle with radius $r$, i.e. $x=\cos \Omega t, y=\sin \Omega t$. Hence,

$r_{2}-r_{1}=-2 k r^{2} C^{2} \approx 2 r^{2} \frac{G M}{R_{0}^{2} c^{2}}=19.66 \mathrm{~mm}$

and it corresponds to the variation of the distance

$$
\Delta r=9.83 \cos D[\mathrm{~mm}]
$$

The variation of the distance (16) is a consequence of the Sun's gravitational potential. The Sun's gravitational potential induces also a much larger tidal perturbation caused by the acceleration from the Sun, but it is well known and the value (13) is free of that tidal effect. The sum of variations of distance (14), (15), and (16) gives

$$
\Delta r=(2.63 \pm 4.1) \cos D[\mathrm{~mm}]
$$

which is very close to the observed value (13). Notice that if the proportionality coefficient is $-2.943 \times 10^{13} \mathrm{~mm}$ [5], then (17) should be replaced by

$$
\Delta r=(2.52 \pm 4.1) \cos D[\mathrm{~mm}]
$$

It differs from the value (13) about $0.28 \mathrm{~mm}$, and it is 20 times smaller than the deviation of the General Relativity theoretical prediction from the observed value.

\section{Conclusion}

We can resume that the measured value (13) is a result of three effects given by (14), (15) and (16). The effect given by (14) is measured in laboratory and so, it must be included in every theory of gravitation. The gravitational effect given by (15) is deduced considering the method shortly presented in section 2 and based on [8]. The effect given by (16) is a specific gravitational effect for gravitationally bound systems, which should be included in any gravitational theory. It is different from the Shapiro time delay, which is already taken into account within (13). In fact, (16) is an 
effect of deformation of the observed Moon's trajectory caused by the Sun's gravitational potential, while the Shapiro time delay of signals does not consider deformation of trajectories, but the delay of signals in different gravitational potentials. Moreover, the effect of Shapiro time delay is usually considered when the two objects (the emitter and the receiver) near a source of gravitation have independent trajectories of one another and have no mutual gravitational interaction. The Earth and the Moon are gravitationally bound, so beside the Shapiro time delay, the effect (16) should also be considered in the theory.

According to this research, the deviation from the observed LLR value (13) is $0.17 \mathrm{~mm}$ (assuming that the proportionality coefficient is $-2.9 \times 10^{13} \mathrm{~mm}$ ) or $0.28 \mathrm{~mm}$ (assuming that the proportionality coefficient is $-2.943 \times 10^{13} \mathrm{~mm}$ ). On the other hand according to the GR which includes only (14), the deviation from (13) is $5.7 \mathrm{~mm}$. This discrepancy is explained in GR as a possible violation of Gravitational WEP as a part of the Strong EP [11], such that the PPN coefficient $\eta$ appears to be non-zero.

\section{References}

[1] Adelberger, E.G., New tests of Einstein's equivalence principle and Newton's inverse-square law. Classical and Quantum Gravity, Vol.18, No.3, (2001), pp. 2397-2405, available online: http://iopscience.iop.org/article/10.1088/0264-9381/18/13/302

[2] Fahnline, D.E., A covariant four-dimensional expression for Lorentz transformations. American Journal of Physics, Vol.50, No.9, (1982), pp. 818-821, http://aapt.scitation.org/doi/abs/10.1119/1.12748

[3] Matolcsi, T., Spacetime without Reference Frames. Akadémiai Kiadó, Budapest, (1993), pp: 156

[4] Nordtvedt, K., Lunar laser ranging, a comprehensive probe of postNewtonian gravity, In: Ciufolini, I. et al (eds.) Gravitation. Villa Mondragone International School of Gravitation and Cosmology, Rome, (2002), pp: 97-113, http://cds.cern.ch/record/599495/files/ 0301024.pdf

[5] Nordtvedt, K. and Vokrouhlicky, D. (1997), Recent progress in analytical modeling of the relativistic effects in the lunar motion. In Wytrzyszczak, I.M., Lieske, J.H. and Feldman, R.A. (eds.) Dynamics and astronomy of the natural and artificial celestial bodies, Kluwer Academic Publishers, Dordrecht, pp. 205-214.

[6] Nordtvedt, K., The relativistic orbit observables in lunar laser ranging. Icarus, Vol.114, (1995), pp. 51-62, https://doi.org/10.1006/icar.1995. 1042

[7] Trenčevski, K., One model of gravitation and mechanics. Tensor, Vol.53, (1993), pp. 70-82

[8] Trenčevski, K., Celakoska, E. and Balan, V., Research of gravitation in flat Minkowski space, International Journal of Theoretical Physics, Vol.50, No.1, (2011), pp. 1-26, doi:10.1007/s10773-010-0488-x.

[9] Trenčevski, K. and Celakoska, E., Equations of motion for two body problem according to an observer inside the gravitational field. Journal of Dynamical Systems \& Geometric Theories Vol.9, No.2, (2011), pp. 115-135, http://dx.doi.org/10.1080/1726037X.2011.10698596.

[10] Trenčevski, K. and Celakoska, E., Representation of geodesics near the Schwarzschild radius. Gravitation and Cosmology Vol.21, No.1, (2014), pp.93-103, doi:10.1134/S0202289315010132.

[11] Will, C.M., Theory and Experiment in Gravitational Physics. Cambridge University Press, New York (1993), pp:185-190.

[12] Will, C.M., The confrontation between General Relativity and Experiment. Living Reviews in Relativity, Vol.9, (2006), pp.3-100. doi: 10.12942/lrr-2014-4

[13] Williams, J.G., Turyshev, S.G. and Boggs, D.H., Lunar laser ranging tests of the Equivalence principle with the Earth and Moon. International Journal of Modern Physics D, Vol.18, (2009), pp. 1129-1175, http://dx.doi.org/10.1142/S021827180901500X.

[14] Williams, J.G., Turyshev, S.G. and Boggs, D.H., Progress in lunar laser ranging tests of relativistic gravity. Physical Reviews Letter Vol.93, (2004), pp. 261101, https://doi.org/10.1103/PhysRevLett.93. 261101

[15] Williams, J.G., Turyshev, S.G. and Murphy, T.W. Jr., Improving LLR tests of gravitational relativity. International Journal of Modern Physics D, Vol. 13, (2004), pp. 567-582, http://dx.doi.org/10.1142/S0218271804004682. 